\title{
Retinal vascular geometry and 6 year incidence and progression of diabetic retinopathy
}

\author{
Carol Yim-lui Cheung ${ }^{1,2}$ • Charumathi Sabanayagam ${ }^{1,3}$ • Antony Kwan-pui Law ${ }^{2}$. \\ Neelam Kumari ${ }^{1}$ • Daniel Shu-wei Ting ${ }^{1} \cdot$ Gavin Tan $^{1} \cdot$ Paul Mitchell ${ }^{4}$ • \\ Ching Yu Cheng ${ }^{1,3,5}$ • Tien Yin Wong ${ }^{1,3,5}$
}

Received: 22 March 2017 / Accepted: 17 May 2017 /Published online: 16 June 2017

(C) Springer-Verlag GmbH Germany 2017

\begin{abstract}
Aims/hypothesis We aimed to examine prospectively the association between a range of retinal vascular geometric variables measured from retinal photographs and the 6 year incidence and progression of diabetic retinopathy.

Methods We conducted a prospective, population-based cohort study of Asian Malay individuals aged 40-80 years at baseline $(n=3280)$ who returned for a 6 year follow-up. Retinal vascular geometric variables (tortuosity, branching, fractal dimension, calibre) were measured from baseline retinal photographs using a computer-assisted program (Singapore I Vessel Assessment). Diabetic retinopathy was graded from baseline and follow-up photographs using the modified Airlie House classification system. Incidence of diabetic retinopathy was defined as a severity of $\geq 15$ at followup among those without diabetic retinopathy at baseline. Incidence of referable diabetic retinopathy was defined as moderate or severe non-proliferative diabetic retinopathy, pro-
\end{abstract}

Tien Yin Wong

wong.tien.yin@snec.com.sg

1 Singapore Eye Research Institute, Singapore National Eye Centre, 11 Third Hospital Avenue, 168751 Singapore, Republic of Singapore

2 Department of Ophthalmology and Visual Sciences, Chinese University of Hong Kong, Hong Kong Special Administrative Region, People's Republic of China

3 Centre for Quantitative Medicine, Duke-NUS Medical School, Singapore, Republic of Singapore

4 Centre for Vision Research, University of Sydney, Sydney, NSW, Australia

5 Ophthalmology and Visual Sciences Academic Clinical Programme, Duke-NUS Medical School, National University of Singapore, Singapore, Republic of Singapore liferative diabetic retinopathy or diabetic macular oedema at follow-up in participants who had had no or mild nonproliferative diabetic retinopathy at baseline. Progression of diabetic retinopathy was defined as an increase in severity of $\geq 2$ steps at follow-up. Log-binomial models with an expectation-maximisation algorithm were used to estimate $\mathrm{RR}$ adjusting for age, sex, diabetes duration, $\mathrm{HbA}_{1 \mathrm{c}}$ level, BP, BMI, estimated GFR and total and HDL-cholesterol at baseline.

Results A total of 427 individuals with diabetes participated in the baseline and 6 year follow-up examinations. Of these, $19.2 \%, 7.57 \%$ and $19.2 \%$ developed incidence of diabetic retinopathy, incidence of referable diabetic retinopathy and diabetic retinopathy progression, respectively. After multivariate adjustment, greater arteriolar simple tortuosity (mean RR [95\% CI], 1.34 [1.04, 1.74]), larger venular branching angle (RR $1.26[1.00,1.59])$ and larger venular branching coefficient (RR $1.26[1.03,1.56])$ were associated with incidence of diabetic retinopathy. Greater arteriolar simple tortuosity (RR $1.82[1.32,2.52])$, larger venular branching coefficient (RR 1.46 [1.03, 2.07]), higher arteriolar fractal dimension (RR $1.59[1.08,2.36])$ and larger arteriolar calibre (RR $1.83[1.15$, 2.90]) were associated with incidence of referable diabetic retinopathy. Greater arteriolar simple tortuosity (RR 1.34 $[1.12,1.61])$ was associated with diabetic retinopathy progression. Addition of retinal vascular variables improved discrimination (C-statistic 0.796 vs $0.733, p=0.031)$ and overall reclassification (net reclassification improvement $18.8 \%$, $p=0.025$ ) of any diabetic retinopathy risk beyond established risk factors.

Conclusions/interpretation Retinal vascular geometry measured from fundus photographs predicted the incidence and progression of diabetic retinopathy in adults with diabetes, beyond established risk factors. 
Keywords Diabetic retinopathy $\cdot$ Epidemiology $\cdot$ Imaging · Retinal vascular geometry

$\begin{array}{ll}\text { Abbreviations } \\ \text { CRAE } & \text { Central retinal artery equivalent } \\ \text { DMO } & \text { Diabetic macular oedema } \\ \text { MABP } & \text { Mean arterial BP } \\ \text { NPDR } & \text { Non-proliferative diabetic retinopathy } \\ \text { NRI } & \text { Net reclassification improvement } \\ \text { PDR } & \text { Proliferative diabetic retinopathy } \\ \text { ROC } & \text { Receiver operating characteristic } \\ \text { SiMES } & \text { Singapore Malay Eye Study } \\ \text { SIVA } & \text { Singapore I Vessel Assessment } \\ \text { VTDR } & \text { Vision-threatening diabetic retinopathy }\end{array}$

\section{Introduction}

Diabetic retinopathy, a leading cause of acquired blindness, is the most frequent and specific diabetes-related microvascular complication $[1,2]$. Early detection of diabetic retinopathy and more severe levels is a key strategy to prevent blindness and visual impairment. In some population-based screening programmes (e.g. UK Singapore), it is useful to predict individuals at risk of 'referable levels of diabetic retinopathy', usually defined as moderate or worse diabetic retinopathy and/or diabetic macular oedema (DMO) [3-5].

It has been suggested that changes in the structure and pattern of the retinal vasculature early in the course of diabetes may be a risk marker of subsequent risk of diabetic retinopathy [6]. In this regard, several studies have reported on the relationship between variation in calibre of the retinal vessels (e.g. wider venules) and severity of diabetic retinopathy [7-9]. However, most studies have been cross-sectional, and the few prospective studies have shown inconsistent results; for example, wider retinal arteriolar calibre was associated with increased risk of diabetic retinopathy in some studies of type 1 $[10,11]$ and type 2 [12-14] diabetes, but not others [15].

According to Murray's principle [16], the branching pattern of vascular networks develops to minimise the energy required to maintain efficient blood flow. Therefore, deviations or alterations from an optimal retinal branching network are considered to result in impaired microcirculatory transport, increased shear stress, reduced efficiency and hence greater risk of vascular damage [6]. Based on this, a range of newer retinal vascular geometric variables (e.g. tortuosity, branching, fractal dimension) have been described. Most studies have explored the relationship between these newer variables and systemic diseases such as stroke and CHD [17-19]. There are few prospective data on whether these variables predict subsequent diabetic retinopathy $[14,20,21]$.
In this study, we prospectively examined the association of retinal vascular geometric variables (tortuosity, branching, fractal dimension) and vessel calibre with 6 year incidence and progression of diabetic retinopathy in a populationbased cohort with type 2 diabetes.

\section{Methods}

\section{Study population}

This was derived from the Singapore Malay Eye Study (SiMES), a population-based study of eye diseases in Asian Malay adults aged 40-80 years at baseline residing in Southwestern Singapore. In brief, participants were selected using an age-stratified (by 10 year age group) random sampling method from a computer-generated list provided by the Singapore Ministry of Home Affairs. Of 4168 eligible participants, 3280 (response rate $78.7 \%$ ) participated in the baseline study, conducted from 2004 through 2006 (SiMES-1). The 6 year follow-up examinations were conducted between 2011 and 2013 (SiMES-2). After excluding 415 individuals who died, 126 who were terminally ill or cognitively impaired, and 103 patients who could not be contacted or had moved away, 1901 of 2636 eligible individuals (response rate, $72.1 \%$ ) participated in SiMES-2. [22].

Participants underwent a standardised interview, systemic and ocular examination, and laboratory investigations at both baseline and follow-up. The study's methodology and objectives have been reported in detail elsewhere [22]. Written informed consent was obtained from each participant; the study conducted adhered to the Declaration of Helsinki. Ethical approval was obtained from the Singapore Eye Research Institute Institutional Review Board.

\section{Definition of diabetes}

Diabetes mellitus was identified if a random plasma glucose was $\geq 11.1 \mathrm{mmol} / 1, \mathrm{HbA}_{1 \mathrm{c}}$ was $\geq 6.5 \%$ (48 $\mathrm{mmol} / \mathrm{mol}$ ) or participants self-reported use of hypoglycaemic medication or had physician-diagnosed diabetes. In this study, participants were considered to have type 2 diabetes if they had been diagnosed as having developed diabetes after 30 years of age.

\section{Assessment of diabetic retinopathy}

Digital retinal fundus photography employed a $45^{\circ}$ digital retinal camera (Canon CR-DGi 10D; Canon, Tokyo, Japan) after pupil dilation using tropicamide $1 \%$ and phenylephrine hydrochloride $2.5 \%$. Two retinal photographs of each eye were obtained, one centred on the optic disc and the other on the fovea. 
Diabetic retinopathy was graded from baseline and followup retinal photographs using the modified Airlie House classification system. Diabetic retinopathy was defined as present if the retinopathy level was $\geq 15$ [23]. The severity score from the worse eye was used for each individual. If the images from one eye were ungradable, the scores for the fellow eye were used to define the outcomes [24]. Severity of diabetic retinopathy was further categorised into minimal non-proliferative diabetic retinopathy (NPDR; level 15-20), mild NPDR (level 35), moderate NPDR (level 43-47), severe NPDR (level 53) and proliferative diabetic retinopathy (PDR, level $\geq 60$ ). DMO was defined as hard exudates in the presence of microaneurysms and blot haemorrhage within one disc diameter from the foveal centre, or presence of focal photocoagulation scars in the macular areas.

We defined two primary endpoints for this study:

- incidence of diabetic retinopathy was defined as a severity of $\geq 15$ at follow-up in participants free of diabetic retinopathy at baseline;

- incidence of referable diabetic retinopathy was defined as newly developed moderate NPDR, severe NPDR, PDR or $\mathrm{DMO}$ at follow-up in those with no diabetic retinopathy, minimal or mild NPDR and no DMO at baseline. This endpoint is clinically useful because, in diabetic retinopathy screening programmes, individuals with referable diabetic retinopathy are referred to eye specialists and ophthalmologists for review, while those with no diabetic retinopathy or minimal or mild diabetic retinopathy continued to be screened in primary care $[3,4]$.

We also defined two secondary endpoints:

- incidence of vision-threatening diabetic retinopathy (VTDR) was defined as newly developed severe NPDR, PDR or DMO at follow-up in participants free of VTDR at baseline:

- the participant's diabetic retinopathy level was derived by concatenating the levels for both eyes, giving the eye with higher diabetic retinopathy level greater weight. This scheme provided a 15 -step scale $(10 / 10,15<15,15 / 15$, $20<20,20 / 20,35<35,35 / 35,43<43,43 / 43,47<47$, $47 / 47,53<53,53 / 53,60+<60+, 60+/ 60+)$ when all levels of PDR were grouped as one level [24]. Progression of diabetic retinopathy was defined as an increase in severity level of $\geq 2$ steps at follow-up in those with steps $10 / 10$ to $53 / 53$ at baseline [24].

\section{Measurements of retinal vascular geometric variables}

We used a semi-automated computer-assisted program (Singapore I Vessel Assessment [SIVA], version 4.0;
National University of Singapore, Singapore) to quantitatively measure a range of retinal vascular geometric variables (tortuosity, branching, fractal dimension) and calibre from baseline optic-disc-centred retinal photographs. SIVA automatically identifies the optic disc, places a grid with reference to its centre, identifies vessel type and calculates retinal vascular variables. Trained graders, masked to participant characteristics, were responsible for visually evaluating SIVA automated measurement and for manual intervention if necessary according to a standardised protocol [19]. The measured area was standardised and defined as the region between 0.5 and 2.0 disc diameters from the disc margin, and all visible vessels coursing through the specified zone were measured (Fig. 1). We used a photograph of each participant's right eye; if this was ungradable, the measurement was performed on the left eye. Intra- and intergrader reliability for measurements were high (CV 0.33-18\%) and have previously been reported [19].

Tortuosity Two measures of vessel tortuosity were used [25, 26]. Simple tortuosity was computed as the actual path length of the vessel segment divided by the straight-line length. Curvature tortuosity was computed as the integral of the curvature square along the path of the vessel, normalised by total path length; this measure is dimensionless as it represents a ratio. Retinal vascular tortuosity reflects the straightness/

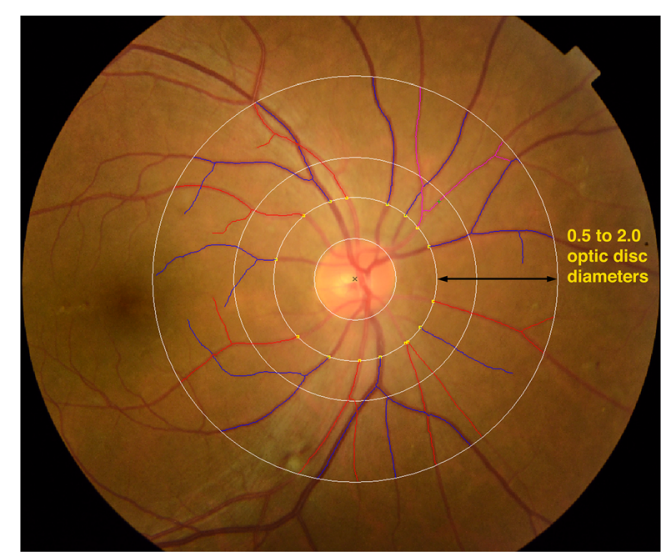

Fig. 1 Measurement of retinal vascular variables (tortuosity, branching, fractal dimension, calibre) from a retinal photograph using SIVA (National University of Singapore, Singapore). SIVA automatically identifies the optic disc, places a grid with reference to its centre, identifies vessel type and calculates retinal variables. The measured areas for retinal vascular variables are standardised as the region from 0.5 to 2.0 optic disc diameters from the disc margin. All the vessels within the measured areas are traced, and an unsupervised approach is used to classify vessels into arterioles or venules. Measurement of retinal variables is performed by trained graders who are responsible for the visual evaluation of SIVA's automated measurements and performing manual intervention if necessary. SIVA's manual options include adjusting grid placement, correcting wrongly identified vessel types, identifying connecting or disconnecting vessel segments (if the measurement segment is considered unreliable) and modifying widths of vessel segments, according to SIVA's grading protocol 
curliness of the vessels; smaller tortuosity values indicate straighter retinal vessels.

Bifurcation The retinal vascular branching angle was defined as the first angle subtended between two daughter vessels at each vascular bifurcation [27]. The mean widths of the parent vessel $\left(\mathrm{d}_{0}\right)$ and the two daughter branching vessels $\left(\mathrm{d}_{1}\right.$ and $\mathrm{d}_{2}$, $\mathrm{d}_{1}<\mathrm{d}_{2}$ ) were also measured. The branching coefficient was defined as the ratio of the branching vessel widths to trunk vessel width $\left(\mathrm{d}_{1}+\mathrm{d}_{2}\right)^{2} / \mathrm{d}_{0}^{2}$ [27]. The retinal branching measurement was not bounded by the standardised measured region (within 0.5 and 2.0 disc diameters). Larger branching coefficient values indicate wider branching vessel widths.

Fractal dimension The retinal vascular fractal dimension was calculated from a skeletonised line tracing using the boxcounting method, and represents a 'global' measure summarising the whole branching pattern of the retinal vascular tree [28]. Larger values indicate a more complex branching pattern.

Calibre Retinal vascular calibre was measured following the standardised protocol used in the Atherosclerosis Risk in Communities (ARIC) study based on the revised KnudtsonParr-Hubbard formula using SIVA. The retinal arteriolar and venular calibres of the large six arterioles and venules were summarised as the central retinal artery equivalent (CRAE) and central retinal vein equivalent, respectively [29].

\section{Assessment of other risk factors}

Age was defined as age at the time of baseline examination. Duration of diabetes was the period between age at diagnosis and age at baseline examination. Systolic and diastolic BPs were measured using a digital automatic blood pressure monitor (Dinamap model Pro Series DP110X-RW, 100V2; GE Medical Systems Information Technologies, Milwaukee, WI, USA), after the participant had been seated for at least $5 \mathrm{~min}$. $\mathrm{BP}$ was measured twice, 5 min apart. A third measurement was made if systolic BP differed by $>10 \mathrm{mmHg}$ or diastolic BP differed by $>5 \mathrm{mmHg}$. The mean between the two closest readings was then taken as the BP of that individual. Mean arterial $\mathrm{BP}$ (MABP) was calculated as two-thirds of the diastolic plus one-third of the systolic BP. Hypertension was defined as systolic BP of $\geq 140 \mathrm{mmHg}$, diastolic blood pressure of $\geq 90 \mathrm{mmHg}$, a self-reported history of physician-diagnosed hypertension or use of antihypertensive medication.

Non-fasting venous blood samples were analysed at the National University Hospital Reference Laboratory for biochemical testing of serum total cholesterol, HDL-cholesterol, LDL-cholesterol, triacylglycerol, $\mathrm{HbA}_{1 \mathrm{c}}$, creatinine and glucose. Current smokers were defined as those currently smoking any number of cigarettes (i.e. current vs past/never).
BMI was calculated as body weight (in kilograms) divided by body height (in meters) squared. eGFR was estimated from standardised serum creatinine using the recently developed Chronic Kidney Disease Epidemiology Collaboration equation.

\section{Statistical analysis}

Independent $t$ test or $\chi^{2}$ tests were used to examine the differences in baseline characteristics between included and excluded participants in SiMES. First, we examined the relation of individual retinal vascular geometric variables measured by SIVA to the four outcomes: incidence of diabetic retinopathy, incidence of referable diabetic retinopathy, incidence of VTDR and progression of diabetic retinopathy, adjusting for established risk factors (age, sex, duration of diabetes, $\mathrm{HbA}_{1 \mathrm{c}}$, MABP, BMI, eGFR, total and HDL-cholesterol) at baseline. Log-binomial models with an expectation-maximisation algorithm were used to estimate the RR and 95\% CI between retinal vascular measures (per each SD increase) and the incidence of diabetic retinopathy, incidence of referable diabetic retinopathy, incidence of VTDR and progression of diabetic retinopathy.

Next, we examined the incremental usefulness of adding retinal measures to the prediction of two primary endpoints: incidence of diabetic retinopathy and incidence of referable diabetic retinopathy. We used principal component analysis to summarise all the outputted SIVA data in this analysis [30] as these would all be included in the retinal image assessment if we used the software for diabetic retinopathy screening. We retained the first to 12th unrotated SIVA components, which explained $85 \%$ of the total variance in the principle component analysis [30]. We calculated the C-statistic for the logbinomial regression models to quantify the predictive discrimination. Differences in C-statistics after addition of SIVA measures to a model with established risk factors were estimated and compared. Second, we assessed the calibration of risk prediction by comparing the predicted frequency of diabetic retinopathy events with the observed frequency, and we calculated the Hosmer-Lemeshow $\chi^{2}$ statistic for the models to test goodness of fit. Values of $p<0.05$ represent a significant difference between the expected and observed event rates, indicating that the model is not well calibrated.

Finally, we examined the net reclassification improvement (NRI) in prediction of incidence of diabetic retinopathy and incidence of referable diabetic retinopathy after adding the SIVA measures. NRI can quantify and evaluate the enhancement in clinically useful risk assessments when new markers are added to a standard risk prediction model. We first generated predicted probabilities for the incidence of diabetic retinopathy and of referable diabetic retinopathy outcomes using established risk factors only. Because no standard risk thresholds exist for populations with diabetes, we tested models of 
the 6 year cumulative incidence of diabetic retinopathy and incidence of referable diabetic retinopathy with two cut-off points (approximately tertiles) at $11.2 \%$ and $19.1 \%$, and $2.0 \%$ and $4.8 \%$, respectively, and classified participants into low-, intermediate- and high-risk categories. We then generated predicted probability for the outcomes using both SIVA measures and established risk factors. We used the same cutoff points, and participants were re-assigned to low-, intermediate- or high-risk categories.

We used $p$ values of $<0.05$ from two-sided tests to indicate statistical significance. All statistical analyses were performed using STATA statistical software version 12 (StataCorp, College Station, TX, USA).

\section{Results}

Of the 3280 participants in SiMES-1, there were 1052 individuals with diabetes. We excluded 625 participants who had no follow-up $(n=559)$, or no $(n=15)$ or ungradable $(n=24)$ retinal images, and individuals with missing data $(n=27)$, leaving 427 persons with diabetes for participation in SiMES-2. Excluded diabetic participants were older and had higher $\mathrm{BP}$, lower $\mathrm{BMI}$ and lower $\mathrm{HbA}_{1 \mathrm{c}}$ than those who were included (Table 1). Of the 308 individuals with no diabetic retinopathy at baseline, $19.2 \%(n=59)$ developed any diabetic retinopathy at follow-up. Of the 383 participants with no referable diabetic retinopathy at baseline, $7.57 \%(n=29)$ developed referable diabetic retinopathy at follow-up. Of the 412 participants with no VTDR at baseline, $2.91 \%(n=12)$ developed VTDR at follow-up (Table 2).

The age- and sex-adjusted relationship between retinal vascular geometric measures and incidence of diabetic retinopathy, incidence of referable diabetic retinopathy, incidence of VTDR and progression of diabetic retinopathy is shown in Table 3. In the multivariate analysis (Table 4), greater simple tortuosity of retinal arterioles (RR per SD increase, mean $[95 \% \mathrm{CI}], 1.34[1.04,1.74])$, larger retinal venular branching angle (RR per SD increase, 1.26 [1.00, 1.59]) and larger venular branching coefficient (RR per SD increase, $1.26[1.03,1.56]$, respectively) were independently associated with incidence of diabetic retinopathy after adjusting for age, sex, duration of diabetes, $\mathrm{HbA}_{1 \mathrm{c}}$, BP, BMI, eGFR and total and HDL-cholesterol levels at baseline. Greater simple tortuosity of retinal arterioles (RR per SD increase, 1.82 [1.32, 2.52]), larger retinal venular branching coefficient (RR per SD increase, $1.46[1.03,2.07])$, higher value of arteriolar fractal dimension (RR per SD increase, 1.59 [1.08, 2.36], respectively) and increased CRAE (RR per SD increase, $1.83[1.15,2.90])$, were independently associated with incidence of referable diabetic retinopathy. Regarding incidence of VTDR, we found that only increased CRAE was independently associated with incidence of VTDR in the age- and sex-adjusted model (Table 3). There were no significant associations between retinal vascular measures and incidence of VTDR after multivariate adjustment, possibly due to the small numbers (Table 4). Of the 416 participants with no diabetic retinopathy or NPDR (steps $10 / 10$ to $53 / 53$ ) at baseline, $19.2 \%(n=80)$ had diabetic retinopathy progression. Only greater simple tortuosity of the retinal arterioles (RR per SD increase $1.34[1.12,1.61]$ ) was independently associated with 6 year progression of diabetic retinopathy after multivariate adjustment (Table 4).

The C-statistic for discrimination of any diabetic retinopathy and referable diabetic retinopathy prediction increased significantly by $8.59 \%$ from 0.733 to $0.796(p=0.031)$ and significantly by $5.40 \%$ from 0.833 to $0.878(p=0.023)$, respectively, after retinal vascular measures were added to the model together with established risk factors (Table 5). Figure 2 shows the receiver operating characteristic (ROC) curves of predictive discrimination for incidence of diabetic retinopathy and referable diabetic retinopathy, respectively. In the calibration assessment, dividing the population at risk into deciles of predicted risk vs observed event rate, the Hosmer-Lemeshow $\chi^{2}$ statistics for incidence of diabetic retinopathy and referable diabetic retinopathy were 12.7 $(p=0.124)$ and $13.4(p=0.098)$ for the basic model, and $9.31(p=0.317)$ and $11.2(p=0.189)$ with the addition of retinal vascular measures, respectively, indicating that neither model had a significant lack of fit. However, retinal vascular measures did not statistically significantly improve the discrimination of VTDR prediction (C-statistics 0.958 vs $0.934, p=0.144)$ and diabetic retinopathy progression (C-statistics 0.764 vs $0.754, p=0.735$ ) (Table 5).

Among participants with no incidence of diabetic retinopathy events (Table 6) or with no referable diabetic retinopathy events (Table 7), 65 and 106 were reclassified to a lower risk category ( $p<0.001$ for both) after addition of retinal vascular measures, respectively. The overall NRI was $18.8 \%$ $(p=0.025)$ and $20.7 \%(p=0.015)$, respectively, indicating that significantly more were being reclassified appropriately than inappropriately.

\section{Discussion}

In our current study, we demonstrated that geometric measures in the retinal vasculature (arteriolar tortuosity, venular branching angle, venular branching coefficient, arteriolar fractal dimension, arteriolar calibre) were associated with risk of diabetic retinopathy in individuals with type 2 diabetes, independent of age, sex, duration of diabetes, $\mathrm{HbA}_{1 \mathrm{c}}$, MABP, BMI, eGFR and total and HDL-cholesterol levels at baseline. We further showed that adding these retinal vascular geometric variables significantly improved discrimination and risk stratification for diabetic retinopathy and referable diabetic 
Table 1 Baseline characteristics of SiMES participants with diabetes

\begin{tabular}{|c|c|c|c|}
\hline Variable & Included $(n=427)$ & Excluded $(n=625)^{\mathrm{a}}$ & $p$ value \\
\hline Age, years & $58.55 \pm 8.66$ & $65.50 \pm 9.70$ & $<0.001$ \\
\hline MABP, mmHg & $102.9 \pm 12.8$ & $106.2 \pm 14.5$ & $<0.001$ \\
\hline BMI, $\mathrm{kg} / \mathrm{m}^{2}$ & $28.4 \pm 4.76$ & $27.13 \pm 4.94$ & $<0.001$ \\
\hline Duration of diabetes, years & $5.11 \pm 6.79$ & $5.96 \pm 8.89$ & 0.094 \\
\hline $\mathrm{HbA}_{1 \mathrm{c}}, \%$ & $8.21 \pm 1.93$ & $7.89 \pm 1.82$ & 0.007 \\
\hline $\mathrm{HbA}_{1 \mathrm{c}}, \mathrm{mmol} / \mathrm{mol}$ & $66.3 \pm 21.1$ & $62.8 \pm 19.9$ & 0.007 \\
\hline Blood glucose, mmol/1 & $10.4 \pm 5.13$ & $9.56 \pm 4.86$ & 0.009 \\
\hline Serum creatinine, mmol/1 & $88.2 \pm 29.4$ & $111.7 \pm 95.0$ & $<0.001$ \\
\hline Total cholesterol, mmol/1 & $5.52 \pm 1.11$ & $5.65 \pm 1.38$ & 0.093 \\
\hline HDL-cholesterol, mmol/1 & $1.28 \pm 0.30$ & $1.27 \pm 0.30$ & 0.610 \\
\hline Triacylglycerol, mmol/1 & $1.97 \pm 0.17$ & $1.96 \pm 0.20$ & 0.286 \\
\hline Retinal arteriolar simple tortuosity & $1.08 \pm 0.02$ & $1.09 \pm 0.02$ & 0.274 \\
\hline Retinal venular simple tortuosity & $1.10 \pm 0.02$ & $1.10 \pm 0.03$ & 0.452 \\
\hline Retinal arteriolar curvature tortuosity $\left(\times 10^{4}\right)$ & $0.463 \pm 0.155$ & $0.501 \pm 0.133$ & 0.332 \\
\hline Retinal venular curvature tortuosity $\left(\times 10^{4}\right)$ & $0.657 \pm 0.168$ & $0.625 \pm 0.268$ & 0.455 \\
\hline Retinal arteriolar branching angle, degrees & $75.4 \pm 11.9$ & $73.4 \pm 24.5$ & 0.635 \\
\hline Retinal venular branching angle, degrees & $78.3 \pm 10.8$ & $80.0 \pm 11.2$ & 0.550 \\
\hline Retinal arteriolar branching coefficient & $1.49 \pm 0.25$ & $1.57 \pm 0.29$ & 0.376 \\
\hline Retinal venular branching coefficient & $1.29 \pm 0.17$ & $1.27 \pm 0.29$ & 0.752 \\
\hline Retinal arteriolar fractal dimension & $1.215 \pm 0.062$ & $1.134 \pm 0.082$ & $<0.001$ \\
\hline Retinal venular fractal dimension & $1.224 \pm 0.052$ & $1.175 \pm 0.089$ & $<0.001$ \\
\hline Retinal arteriolar calibre (CRAE), $\mu \mathrm{m}$ & $141.0 \pm 11.4$ & $137.1 \pm 15.4$ & 0.185 \\
\hline Retinal venular calibre (CRVE), $\mu \mathrm{m}$ & $209.3 \pm 18.0$ & $209.4 \pm 22.2$ & 0.990 \\
\hline Sex, male & $186(43.6)$ & $298(47.7)$ & 0.052 \\
\hline Hypoglycaemic medication, yes & $251(58.8)$ & $311(49.8)$ & 0.038 \\
\hline Antihyperlipidaemia medication, yes & $141(33.0)$ & 179 (28.6) & 0.307 \\
\hline Antihypertensive medication, yes & $198(46.4)$ & $309(49.4)$ & 0.091 \\
\hline Current smoking, yes & $67(15.7)$ & $89(14.2)$ & 0.751 \\
\hline
\end{tabular}

Data are mean $\pm \mathrm{SD}$ or $n(\%)$

${ }^{\text {a }}$ Excluded due to lack of follow-up, missing retinal photographs from either SiMES-1 or SiMES-2, ungradable photographs or missing covariates retinopathy. Our findings support the concept that computer software assessment of the retinal vasculature in fundus photographs may provide a means to risk stratify individuals at risk of diabetic retinopathy.
Most previous studies examining the relationship between retinal vascular geometric variables have been cross-sectional, and the few prospective studies have shown inconsistent results. In the older population-based sample with type 2
Table 2 Six year cumulative incidence of diabetic retinopathy, incidence of referable diabetic retinopathy, incidence of VTDR and progression of diabetic retinopathy in SiMES

\begin{tabular}{lllc}
\hline Endpoint & No. at risk & No. of events & Incidence (\%) \\
\hline Incidence of diabetic retinopathy & $308^{\mathrm{a}}$ & 59 & 19.2 \\
Incidence of referable diabetic retinopathy & $383^{\mathrm{b}}$ & 29 & 7.57 \\
Incidence of VTDR & $412^{\mathrm{c}}$ & 12 & 2.91 \\
Two-step diabetic retinopathy progression & $416^{\mathrm{d}}$ & 80 & 19.2 \\
\hline
\end{tabular}

${ }^{\text {a }}$ Participants with no diabetic retinopathy at baseline

${ }^{\mathrm{b}}$ Participants with no referable diabetic retinopathy at baseline

${ }^{\mathrm{c}}$ Participants with no VTDR at baseline

${ }^{\mathrm{d}}$ Participants with concatenated levels from 10/10 to 53/53 at baseline 
Table 3 Age- and sex-adjusted relationships between retinal vascular measures (per SD increase) and incidence of diabetic retinopathy, incidence of VTDR and diabetic retinopathy progression in SiMES

\begin{tabular}{|c|c|c|c|c|c|c|c|c|}
\hline \multirow[t]{2}{*}{ Variable } & \multicolumn{2}{|c|}{$\begin{array}{l}\text { Any diabetic retinopathy } \\
(59 / 308)\end{array}$} & \multicolumn{2}{|c|}{$\begin{array}{l}\text { Referable diabetic } \\
\text { retinopathy }(28 / 383)\end{array}$} & \multicolumn{2}{|l|}{ VTDR $(12 / 412)$} & \multicolumn{2}{|c|}{$\begin{array}{l}\text { Diabetic retinopathy } \\
\text { progression }(80 / 416)\end{array}$} \\
\hline & $\mathrm{RR}(95 \% \mathrm{CI})$ & $p$ value & $\mathrm{RR}(95 \% \mathrm{CI})$ & $p$ value & $\mathrm{RR}(95 \% \mathrm{CI})$ & $p$ value & $\mathrm{RR}(95 \% \mathrm{CI})$ & $p$ value \\
\hline Retinal arteriolar simple tortuosity & $1.24(1.01,1.52)$ & 0.043 & $1.38(1.06,1.80)$ & 0.016 & $0.91(0.49,1.70)$ & 0.773 & $1.22(1.03,1.44)$ & 0.022 \\
\hline Retinal venular simple tortuosity & $1.13(0.92,1.38)$ & 0.254 & $0.97(0.68,1.38)$ & 0.846 & $0.74(0.39,1.40)$ & 0.348 & $1.05(0.87,1.27)$ & 0.624 \\
\hline Retinal arteriolar curvature tortuosity & $0.98(0.78,1.24)$ & 0.892 & $1.10(0.90,1.34)$ & 0.345 & $0.82(0.33,2.06)$ & 0.667 & $1.03(0.89,1.18)$ & 0.705 \\
\hline Retinal venular curvature tortuosity & $1.04(0.87,1.24)$ & 0.679 & $1.00(0.70,1.43)$ & 0.995 & $0.49(0.21,1.15)$ & 0.100 & $1.04(0.88,1.22)$ & 0.647 \\
\hline Retinal arteriolar branching angle & $0.99(0.79,1.25)$ & 0.947 & $1.39(0.98,1.98)$ & 0.067 & $0.86(0.51,1.47)$ & 0.581 & $1.06(0.87,1.29)$ & 0.574 \\
\hline Retinal venular branching angle & $1.17(0.93,1.48)$ & 0.183 & $0.86(0.61,1.21)$ & 0.386 & $0.74(0.44,1.24)$ & 0.255 & $1.09(0.89,1.33)$ & 0.411 \\
\hline Retinal arteriolar branching coefficient & $1.02(0.81,1.29)$ & 0.869 & $0.96(0.67,1.36)$ & 0.803 & $1.12(0.69,1.83)$ & 0.645 & $1.02(0.85,1.24)$ & 0.809 \\
\hline Retinal venular branching coefficient & $1.16(0.95,1.41)$ & 0.144 & $1.19(0.89,1.59)$ & 0.244 & $1.08(0.65,1.80)$ & 0.757 & $1.12(0.94,1.35)$ & 0.215 \\
\hline Retinal arteriolar fractal dimension & $1.12(0.87,1.43)$ & 0.390 & $1.61(1.10,2.34)$ & 0.013 & $0.95(0.53,1.69)$ & 0.859 & $1.13(0.91,1.39)$ & 0.267 \\
\hline Retinal venular fractal dimension & $0.82(0.63,1.06)$ & 0.124 & $1.25(0.85,1.84)$ & 0.254 & $0.94(0.53,1.66)$ & 0.819 & $1.02(0.82,1.27)$ & 0.833 \\
\hline Retinal arteriolar calibre (CRAE) & $1.27(0.97,1.67)$ & 0.084 & $1.67(1.12,2.48)$ & 0.012 & $2.22(1.19,4.13)$ & 0.012 & $1.11(0.88,1.41)$ & 0.373 \\
\hline Retinal venular calibre (CRVE) & $0.83(0.63,1.10)$ & 0.197 & $0.91(0.59,1.42)$ & 0.692 & $0.78(0.40,1.50)$ & 0.454 & $1.00(0.78,1.28)$ & 0.989 \\
\hline
\end{tabular}

All groups were adjusted for age and sex at baseline

diabetes in our study, retinal arteriolar tortuosity, a measure of the undulation of a vessel along its course, was associated with increased risk of any diabetic retinopathy, referable diabetic retinopathy and 6 year progression of diabetic retinopathy. Our study extends the observations in a few studies in younger individuals with type 1 diabetes that also show greater retinal arteriolar tortuosity associated with diabetic retinopathy, adverse diabetic risk profile (higher $\mathrm{HbA}_{1 \mathrm{c}}$ and systolic $\mathrm{BP}$ ) and incidence of diabetic retinopathy [31-33]. The underlying mechanism(s) for this observation are less clear. Some researchers have suggested that retinal vascular tortuosity is related to diabetes-related haemodynamic changes such as disturbed blood flow and endothelial dysfunction [34-36]. Greater vessel tortuosity may also reflect increased angiogenesis and increased production of vascular endothelial growth factor [37], so that greater tortuosity may also be interpreted as

Table 4 Multivariate adjusted relationships between retinal vascular measures (per each SD increase) and incidence of diabetic retinopathy, incidence of VTDR and diabetic retinopathy progression in SiMES

\begin{tabular}{|c|c|c|c|c|c|c|c|c|}
\hline \multirow[t]{2}{*}{ Variable } & \multicolumn{2}{|c|}{$\begin{array}{l}\text { Any diabetic retinopathy } \\
(59 / 308)\end{array}$} & \multicolumn{2}{|c|}{$\begin{array}{l}\text { Referable diabetic } \\
\text { retinopathy }(28 / 383)\end{array}$} & \multicolumn{2}{|l|}{ VTDR (12/412) } & \multicolumn{2}{|c|}{$\begin{array}{l}\text { Diabetic retinopathy } \\
\text { progression }(80 / 416)\end{array}$} \\
\hline & $\mathrm{RR}$ & & ) & value & $\mathrm{RR}(95 \% \mathrm{CI})$ & & $\mathrm{RR}(95 \% \mathrm{CI})$ & (at \\
\hline 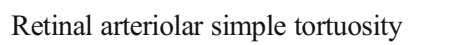 & $(1.04,1.74)$ & 0 & $2(1.32,2.52)$ & $<0.001$ & $5(0.61,2.20)$ & 0.664 & $1.34(1.12,1.61)$ & 0.002 \\
\hline etinal venular simple tortuosity & $1.03(0.83,1.30)$ & 0.775 & $38(0.59,1.31)$ & 0.526 & $0.65(0.32,1.34)$ & 0.241 & $0.99(0.81,1.21)$ & 0.903 \\
\hline etinal arteriolar curvature tortuosity & $1.00(0.79,1.26)$ & 0.973 & $14(0.96,1.36)$ & 0.131 & $1.02(0.48,2.20)$ & 0.957 & $1.04(0.91,1.20)$ & 0.534 \\
\hline etinal venular curvature tortuosity & $1.08(0.94,1.25)$ & 0.290 & $1.11(0.81,1.52)$ & 0.526 & $0.54(0.23,1.28)$ & 0.161 & $1.07(0.92,1.24)$ & 0.399 \\
\hline etinal arteriolar branching angle & $0.97(0.76,1.26)$ & 0.842 & $1.18(0.84,1.66)$ & 0.337 & $0.86(0.49,1.50)$ & 0.589 & $0.98(0.80,1.19)$ & 0.804 \\
\hline Retinal venular branching angle & $1.26(1.00,1.59)$ & 0.045 & $0.89(0.62,1.28)$ & 0.538 & $0.86(0.51,1.45)$ & 0.568 & $1.16(0.94,1.42)$ & 0.161 \\
\hline etinal arteriolar branching coefficient & $0.98(0.80,1.21)$ & 0.853 & $0.90(0.67,1.22)$ & 0.504 & $0.89(0.57,1.39)$ & 0.605 & $0.99(0.84,1.17)$ & 0.932 \\
\hline Retinal venular branching coefficient & $1.26(1.03,1.56)$ & 0.029 & $1.46(1.03,2.07)$ & 0.035 & $1.29(0.76,2.19)$ & 0.347 & $1.16(0.97,1.39)$ & 0.102 \\
\hline Retinal arteriolar fractal d & $1.11(0.86,1.43)$ & 0.441 & $1.59(1.08,2.36)$ & 0.020 & $0.95(0.56,1.59)$ & 0.831 & $1.13(0.92,1.38)$ & 0.250 \\
\hline lar fractal dimension & $0.83(0.65,1.07)$ & 0.156 & $1.44(0.93,2.23)$ & 0.102 & $1.13(0.67,1.90)$ & 0.660 & $1.14(0.91,1.42)$ & 0.264 \\
\hline Retinal arteriolar calibre (CRAE) & $1.02(0.80,1.30)$ & 0.858 & $1.83(1.15,2.90)$ & 0.010 & $1.46(0.77,2.78)$ & 0.252 & $0.99(0.81,1.20)$ & 0.879 \\
\hline Retinal venular calibre (CRVE) & $0.85(0.71,1.01)$ & 0.058 & $0.77(0.47,1.25)$ & 0.295 & $0.92(0.45,1.92)$ & 0.832 & $0.92(0.76,1.11)$ & 0.390 \\
\hline
\end{tabular}

All groups were adjusted for age, sex, MABP, BMI, diabetes duration, $\mathrm{HbA}_{1 \mathrm{c}}$, total cholesterol, HDL-cholesterol and eGFR at baseline 
Table 5 Predictive discrimination for incidence of diabetic retinopathy and VTDR, and progression of diabetic retinopathy

\begin{tabular}{llcc}
\hline Group & Model & C-statistic & $p$ value $^{\mathrm{a}}$ \\
\hline Any diabetic retinopathy & Established risk factors & 0.733 & Referent \\
& Established risk factors + retinal vascular measures & 0.796 & 0.031 \\
Referable diabetic retinopathy & Established risk factors & 0.833 & Referent \\
& Established risk factors + retinal vascular measures & 0.878 & 0.023 \\
VTDR & Established risk factors & 0.934 & Referent \\
& Established risk factors + retinal vascular measures & 0.958 & 0.144 \\
Diabetic retinopathy & Established risk factors & 0.754 & Referent \\
progression & Established risk factors + retinal vascular measures & 0.764 & 0.735 \\
\hline
\end{tabular}

Established risk factors included age, sex, MABP, BMI, duration of diabetes, $\mathrm{HbA}_{1 \mathrm{c}}$, total cholesterol, HDLcholesterol and eGFR at baseline

${ }^{\mathrm{a}} p$ value is for the comparison with the model with established risk factors a very early sign of the neovascularisation seen in later stages of diabetic retinopathy. Greater arteriolar tortuosity may also indicate impaired arteriolar autoregulation [38], leading to capillary wall dilatation (microaneurysm), leakage (oedema, hard exudates) and rupture (haemorrhage) [2].

Similarly, in our study, wider venular branching angle and increased venular branching coefficient, which are measures of circulatory efficiency and optimality, were associated with increased risk of diabetic retinopathy. Efficiency of blood flow is reduced when the bifurcation deviates from the optimal retinal vascular network [16, 27]. Our finding that a deviated venular bifurcation was associated with incidence of diabetic retinopathy may reflect alterations in blood flow associated with the development of diabetic retinopathy. In addition, studies have shown that wider retinal venular calibre is associated with markers of inflammation and endothelial dysfunction such as C-reactive protein and soluble intercellular adhesion molecule-1 [39-41]. Furthermore, a recent study has reported that an increased retinal venular branching coefficient is associated with higher risk of neuropathy, another diabetic microvascular complication, in participants with type 1 diabetes [42]. Therefore, in addition to disturbed blood flow, an increased venular branching coefficient, indicating wider venular branching width, may also reflect inflammation and endothelial dysfunction triggered by hyperglycaemia in diabetes, which has been suggested to be involved in the pathophysiology of diabetic retinopathy [43].

Most previous studies have examined variations in retinal vascular calibre and suggested that specifically wider venular calibre may be a risk marker of diabetic retinopathy and may be useful to predict the incidence and progression of diabetic retinopathy [44]. However, these findings have been largely reported from cross-sectional studies, and data from prospective studies on the relationship between retinal vascular calibre and incidence and progression of diabetic retinopathy are limited, with inconsistent findings [44]. In this study, we found that wider retinal arteriolar, but not venular, calibre, was related to incidence of referable diabetic retinopathy, consistent with the few previous prospective studies [10-14, 45]. For example, wider arteriolar calibre was associated with incidence of diabetic retinopathy in both type $1[10,11]$ and type $2[12,13]$ diabetes in Australia, similar to our current findings. In another study, data from a group with type 1 diabetes in the Wisconsin Epidemiologic Study of Diabetic Retinopathy (WESDR) showed that wider retinal arteriolar calibre was associated with progression of diabetic retinopathy [45]. Our findings further support that arteriolar dilation is a sign of impaired arteriolar autoregulation due to hyperglycaemia-mediated endothelin-1 resistance and calcium influx channel inhibition in smooth muscle cells, which has been suggested to play a pivotal role in the initiation and progression of diabetic retinopathy $[10,38]$. In contrast, in young individuals with type 1 diabetes in Denmark, narrower arteriolar calibre was found to be associated with 16 year incidence of PDR [21]. Furthermore, some studies have shown that neither arteriolar nor venular calibres were related to development of diabetic retinopathy [15], whereas others have found that wider venular, but not arteriolar, calibre is associated with an increased risk of progression of diabetic retinopathy $[45,46]$. The reasons for these discrepancies are unknown and probably reflect variations in age, sample size, follow-up periods, cardiovascular risk factors and type of diabetes that limit direct comparison.

In addition to retinal vascular calibre, another retinal vascular measure - fractal dimension - has been reported to be associated with prevalence of diabetic retinopathy [47] and incidence of PDR in type 1 diabetes [21]. Similar to the association between arteriolar calibre and incidence of referable diabetic retinopathy, we found that higher values of arteriolar fractal dimension, a global measure of complexity of the 

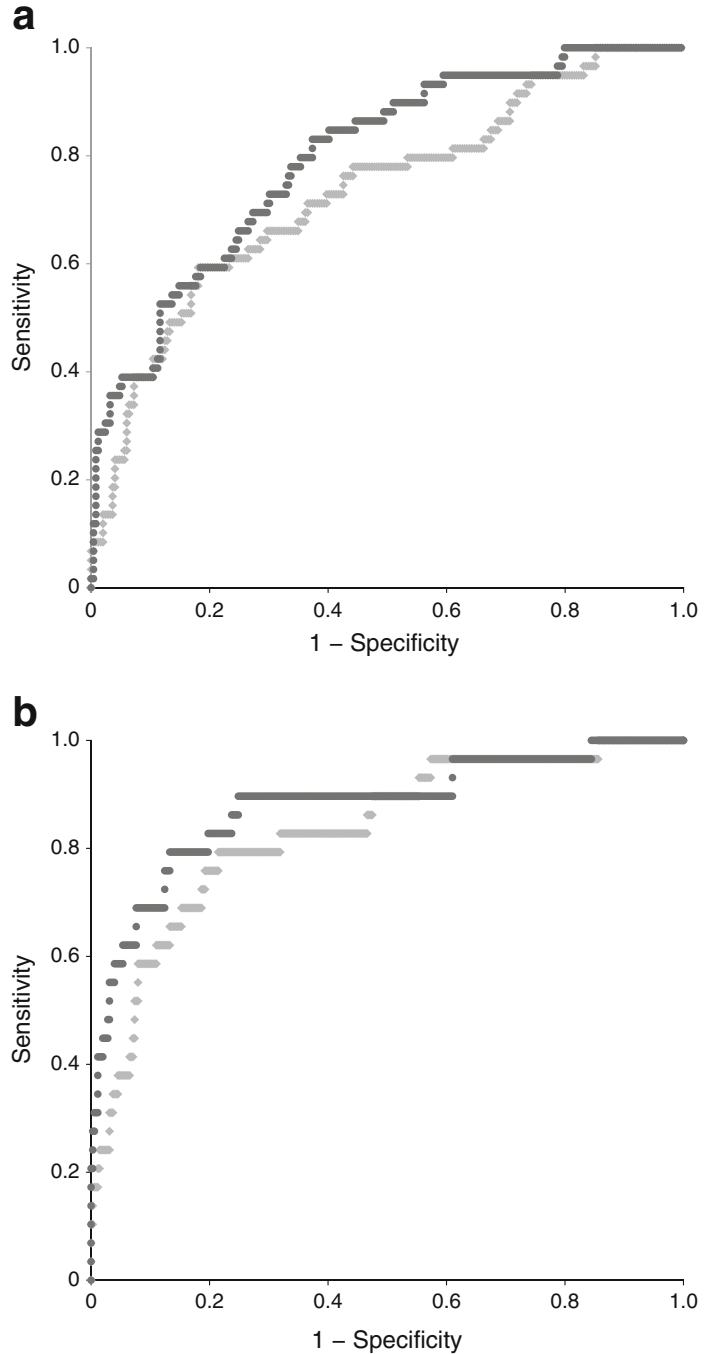

Fig. 2 ROC curves of predictive discrimination for (a) incidence of diabetic retinopathy and (b) incidence of referable diabetic retinopathy. Light grey, first model based on established risk factors only; dark grey, second model based on retinal vascular measures and established risk factors. After retinal vascular measures were added to the model with established risk factors, the $\mathrm{C}$-statistic for discrimination of any diabetic retinopathy prediction increased significantly by approximately $9 \%$ ( $p=0.031$ ), from 0.733 to 0.796 , and the $\mathrm{C}$-statistic for discrimination of referable diabetic retinopathy prediction increased significantly by approximately $5 \%$ ( $p=0.023)$, from 0.833 to 0.878

arteriolar branching pattern, were associated with incidence of referable diabetic retinopathy in our longitudinal data. In contrast, Lim et al found no association between retinal vascular fractal dimension and incidence of diabetic retinopathy in type 1 diabetes [20]. Therefore, caution must be applied in interpreting the findings.

It is well known that traditional risk factors (e.g. $\mathrm{HbA}_{1 \mathrm{c}}$, $\mathrm{BP}$, duration of diabetes) do not fully explain the risk of an individual developing diabetic retinopathy [48]. We showed that the addition of retinal vascular measures improved discrimination and reclassification for diabetic retinopathy, particularly in reclassifying participants without any incidence of diabetic retinopathy or referable diabetic retinopathy to a lower risk category, compared with models using only established risk factors. This suggests that retinal vascular geometric measures provide additional information in identifying individuals who are at low/high risk of diabetic retinopathy. How might this information be useful? Currently, individuals with diabetes are in many countries recommended to undergo annual fundus photography for diabetic retinopathy screening [1]. Incorporating retinal vascular assessment might thus allow a more 'personalised approach' to screening intervals; for example, those with lower risk may need only 2 -yearly screening $[49,50]$. Such approaches need to undergo further validation and cost-effectiveness analysis.

Strengths of our study include the low refusal rate and use of standardised protocols for diabetic retinopathy assessment and retinal vasculature measurement with a computer-assisted program. There are, however, several limitations. First, most participants showing any incidence of disease being measured had NPDR; only 12 had incident VTDR. Therefore, it remains unclear whether retinal vascular measures could predict the risk of more severe diabetic retinopathy. Second, serum glucose and lipids were not measured from fasting venous samples, limiting the precision of these measures. Third, we did not collect seven-field stereoscopic retinal photographs to assess diabetic retinopathy; thus, mild disease may have been missed or misclassified. Nevertheless, using two-field retinal photographs reflects the actual situation for diabetic retinopathy screening. Fourth, $60 \%$ of our participants from SiMES-1 were excluded from the final analysis, so the generalisability of our study results may be limited. Further replication is needed. Fifth, there is currently no definite threshold of retinal vascular variables that can identify individuals at risk as retinal vascular variables overlap between 'disease' and 'no disease'. Assessment of changes in retinal vessel variables over time at an individual level is a better approach to identify individuals at risk. Ongoing studies are required to evaluate this approach. For example, we have started a national screening programme for diabetic retinopathy in Singapore based on retinal photography and telemedicine transmission of images to a central reading centre [5]. We are currently testing our software in this programme to help refine individual risk prediction for diabetic retinopathy screening. Sixth, the retinal vascular variables were measured by a semi-automated computer-assisted program, which may introduce variability between graders and limit application in clinical settings. Newer fully automated software is currently being developed [51, 52]. We are also developing 'deep learning' techniques using artificial intelligence, but this requires extremely large sample sizes, as recently demonstrated for automated diabetic retinopathy detection [53, 54]. Replicating our results and shifting towards measurement using this fully automated artificial intelligence software are the next steps in this research. 
Table 6 Reclassification of diabetic retinopathy risk after addition of retinal vascular measures in the prediction models of incidence of any diabetic retinopathy

\begin{tabular}{|c|c|c|c|c|c|c|c|c|}
\hline \multirow[t]{2}{*}{ Group } & \multicolumn{4}{|c|}{ Retinal vascular measures + established risk category } & \multirow{2}{*}{$\begin{array}{l}\text { Reclassified as } \\
\text { higher risk }(n)\end{array}$} & \multirow{2}{*}{$\begin{array}{l}\text { Reclassified as } \\
\text { lower risk }(n)\end{array}$} & \multirow[t]{2}{*}{ NRI $(\%)$} & \multirow[t]{2}{*}{$p$ value } \\
\hline & Low $(n)$ & Intermediate $(n)$ & $\operatorname{High}(n)$ & Total $(n)$ & & & & \\
\hline \multicolumn{9}{|c|}{ Participants with incident diabetic retinopathy } \\
\hline Established risk category & & & & & 11 & 8 & 5.08 & 0.491 \\
\hline Low & 3 & 5 & 3 & 11 & & & & \\
\hline Intermediate & 4 & 4 & 3 & 11 & & & & \\
\hline High & 1 & 3 & 33 & 37 & & & & \\
\hline Total & 8 & 12 & 39 & 59 & & & & \\
\hline \multicolumn{9}{|c|}{ Participants with no incident diabetic retinopathy } \\
\hline Established risk category & & & & & 31 & 65 & 13.7 & $<0.001$ \\
\hline Low & 76 & 11 & 4 & 91 & & & & \\
\hline Intermediate & 44 & 32 & 16 & 92 & & & & \\
\hline High & 9 & 12 & 45 & 66 & & & & \\
\hline Total & 129 & 55 & 65 & 249 & & & & \\
\hline NRI, \% & & & & & & & 18.8 & 0.025 \\
\hline
\end{tabular}

Risk categories defined by approximate tertiles: low, 0-11.2\%; intermediate, $11.3-19.1 \%$; high, $>19.1 \%$

Established risk factors include age, sex, MABP, BMI, duration of diabetes, $\mathrm{HbA}_{1 \mathrm{c}}$, total cholesterol, HDL-cholesterol and eGFR at baseline

In conclusion, our study shows that quantitative assessment of geometric measures of retinal vasculature provides prognostic information on the risk of diabetic retinopathy and referable diabetic retinopathy in individuals with type 2 diabetes. Adding such retinal vascular measures improves risk discrimination and stratification of diabetic retinopathy beyond standard systemic risk factors. These findings highlight the potential usefulness of examining retinal photographs in screening programmes to improve risk stratification for people with diabetes.

Table 7 Reclassification of diabetic retinopathy risk after addition of retinal vascular measures in the prediction models of incidence of referable diabetic retinopathy

\begin{tabular}{|c|c|c|c|c|c|c|c|c|}
\hline \multirow[t]{2}{*}{ Group } & \multicolumn{4}{|c|}{ Retinal vascular measures + established risk category } & \multirow{2}{*}{$\begin{array}{l}\text { Reclassified as } \\
\text { higher risk }(n)\end{array}$} & \multirow{2}{*}{$\begin{array}{l}\text { Reclassified as } \\
\text { lower risk }(n)\end{array}$} & \multirow[t]{2}{*}{ NRI (\%) } & \multirow[t]{2}{*}{$p$ value } \\
\hline & Low $(n)$ & Intermediate $(n)$ & $\operatorname{High}(n)$ & Total $(n)$ & & & & \\
\hline \multicolumn{9}{|c|}{ Participants with incident diabetic retinopathy } \\
\hline Established risk category & & & & & 3 & 2 & $3.45 \%$ & 0.655 \\
\hline Low & 1 & 0 & 0 & 1 & & & & \\
\hline Intermediate & 2 & 0 & 3 & 5 & & & & \\
\hline High & 0 & 0 & 23 & 23 & & & & \\
\hline Total & 3 & 0 & 26 & 29 & & & & \\
\hline \multicolumn{9}{|c|}{ Participants with no incident diabetic retinopathy } \\
\hline Established risk category & & & & & 45 & 106 & $17.2 \%$ & $<0.001$ \\
\hline Low & 102 & 19 & 5 & 126 & & & & \\
\hline Intermediate & 65 & 37 & 21 & 123 & & & & \\
\hline High & 18 & 23 & 64 & 105 & & & & \\
\hline Total & 185 & 79 & 90 & 354 & & & & \\
\hline NRI, \% & & & & & & & $20.7 \%$ & 0.015 \\
\hline
\end{tabular}

Risk categories defined by approximate tertiles: low, 0-2.0\%; intermediate, $2.1-4.8 \%$; high, $>4.8 \%$

Established risk factors include age, sex, MABP, BMI, duration of diabetes, $\mathrm{HbA}_{1 \mathrm{c}}$, total cholesterol, HDL-cholesterol and eGFR at baseline 
Acknowledgements The authors thank the staff and participants of the SiMES study for their important contributions.

Data availability Data are available on request from the authors.

Funding The authors would like to acknowledge the National Medical Research Council (NMRC) Singapore (grant numbers 0796/2003, 1249/2010 and STaR/0016/2013) for funding support.

Duality of interest The authors declare that there is no duality of interest associated with this manuscript.

Contribution statement Conception and design were carried out by CYCheung, CYCheng and TYW. Data were acquired by CS and NK. The data were analysed and interpreted by CYCheung, AKL, DST, GT and PM. The article was drafted or critically revised for important intellectual content by CYCheung, CS, AKL, NK, DST, GT, PM, CYCheng and TYW. Final approval of the version to be published was given by CYCheung, CS, AKL, NK, DST, GT, PM, CYCheng and TYW. CYCheung is the guarantor of this work.

\section{References}

1. Wong TY, Cheung CM, Larsen M, Sharma S, Simo R (2016) Diabetic retinopathy. Nat Rev Dis Primers 2:16012

2. Cheung N, Mitchell P, Wong TY (2010) Diabetic retinopathy. Lancet 376:124-136

3. Scanlon PH, Malhotra R, Thomas G et al (2003) The effectiveness of screening for diabetic retinopathy by digital imaging photography and technician ophthalmoscopy. Diabet Med 20:467-474

4. Thomas RL, Dunstan F, Luzio SD et al (2012) Incidence of diabetic retinopathy in people with type 2 diabetes mellitus attending the Diabetic Retinopathy Screening Service for Wales: retrospective analysis. BMJ 344:e874

5. Nguyen HV, Tan GS, Tapp RJ et al (2016) Cost-effectiveness of a National Telemedicine Diabetic Retinopathy Screening Program in Singapore. Ophthalmology 123:2571-2580

6. Cheung CY, Ikram MK, Klein R, Wong TY (2015) The clinical implications of recent studies on the structure and function of the retinal microvasculature in diabetes. Diabetologia 58:871-885

7. Kifley A, Wang JJ, Cugati S, Wong TY, Mitchell P (2007) Retinal vascular caliber, diabetes, and retinopathy. Am J Ophthalmol 143: 1024-1026

8. Tsai AS, Wong TY, Lavanya R et al (2011) Differential association of retinal arteriolar and venular caliber with diabetes and retinopathy. Diabetes Res Clin Pract 94:291-298

9. Nguyen TT, Wang JJ, Sharrett AR et al (2008) Relationship of retinal vascular caliber with diabetes and retinopathy: the multiethnic study of atherosclerosis (MESA). Diabetes Care 31:544-549

10. Cheung N, Rogers SL, Donaghue KC, Jenkins AJ, Tikellis G, Wong TY (2008) Retinal arteriolar dilation predicts retinopathy in adolescents with type 1 diabetes. Diabetes Care 31:1842-1846

11. Alibrahim E, Donaghue KC, Rogers S et al (2006) Retinal vascular caliber and risk of retinopathy in young patients with type 1 diabetes. Ophthalmology 113:1499-1503

12. Rogers SL, Tikellis G, Cheung N et al (2008) Retinal arteriolar caliber predicts incident retinopathy: the Australian Diabetes, Obesity and Lifestyle (AusDiab) study. Diabetes Care 31:761-763

13. Cheung N, Tikellis G, Wang JJ (2007) Diabetic retinopathy. Ophthalmology 114:2098-2099 author reply 2099
14. Crosby-Nwaobi R, Heng LZ, Sivaprasad S (2012) Retinal vascular calibre, geometry and progression of diabetic retinopathy in type 2 diabetes mellitus. Ophthalmologica 228:84-92

15. Klein R, Klein BE, Moss SE, Wong TY (2007) Retinal vessel caliber and microvascular and macrovascular disease in type 2 diabetes: XXI: the Wisconsin Epidemiologic Study of Diabetic Retinopathy. Ophthalmology 114:1884-1892

16. Murray CD (1926) The physiological principle of minimum work: I. The vascular system and the cost of blood volume. Proc Natl Acad Sci U S A 12:207-214

17. Hughes AD, Wong TY, Witt $N$ et al (2009) Determinants of retinal microvascular architecture in normal subjects. Microcirculation 16: $159-166$

18. Doubal FN, MacGillivray TJ, Patton N, Dhillon B, Dennis MS, Wardlaw JM (2010) Fractal analysis of retinal vessels suggests that a distinct vasculopathy causes lacunar stroke. Neurology 74:11021107

19. Cheung CY, Tay WT, Mitchell P et al (2011) Quantitative and qualitative retinal microvascular characteristics and blood pressure. J Hypertens 29:1380-1391

20. Lim SW, Cheung N, Wang JJ et al (2009) Retinal vascular fractal dimension and risk of early diabetic retinopathy: a prospective study of children and adolescents with type 1 diabetes. Diabetes Care 32:2081-2083

21. Broe R, Rasmussen ML, Frydkjaer-Olsen U et al (2014) Retinal vessel calibers predict long-term microvascular complications in type 1 diabetes: the Danish Cohort of Pediatric Diabetes 1987 (DCPD1987). Diabetes 63:3906-3914

22. Rosman M, Zheng Y, Wong W et al (2012) Singapore Malay Eye Study: rationale and methodology of 6-year follow-up study (SiMES-2). Clin Exp Ophthalmol 40:557-568

23. Wong TY, Cheung N, Tay WT et al (2008) Prevalence and risk factors for diabetic retinopathy: the Singapore Malay Eye Study. Ophthalmology 115:1869-1875

24. Klein R, Knudtson MD, Lee KE, Gangnon R, Klein BE (2008) The Wisconsin Epidemiologic Study of Diabetic Retinopathy: XXII the twenty-five-year progression of retinopathy in persons with type 1 diabetes. Ophthalmology 115:1859-1868

25. Witt N, Wong TY, Hughes AD et al (2006) Abnormalities of retinal microvascular structure and risk of mortality from ischemic heart disease and stroke. Hypertension 47:975-981

26. Cheung CY, Zheng Y, Hsu W et al (2011) Retinal vascular tortuosity, blood pressure, and cardiovascular risk factors. Ophthalmology 118:812-818

27. Zamir M, Medeiros JA, Cunningham TK (1979) Arterial bifurcations in the human retina. J Gen Physiol 74:537-548

28. Cheung CY, Thomas GN, Tay W et al (2012) Retinal vascular fractal dimension and its relationship with cardiovascular and ocular risk factors. Am J Ophthalmol 154(663-674):e661

29. Cheung CY, Hsu W, Lee ML et al (2010) A new method to measure peripheral retinal vascular caliber over an extended area. Microcirculation 17:495-503

30. Cheung CY, Tay WT, Ikram MK et al (2013) Retinal microvascular changes and risk of stroke: the Singapore Malay Eye Study. Stroke 44:2402-2408

31. Sasongko MB, Wang JJ, Donaghue KC et al (2010) Alterations in retinal microvascular geometry in young type 1 diabetes. Diabetes Care 33:1331-1336

32. Benitez-Aguirre P, Craig ME, Sasongko MB et al (2011) Retinal vascular geometry predicts incident retinopathy in young people with type 1 diabetes: a prospective cohort study from adolescence. Diabetes Care 34:1622-1627

33. Sasongko MB, Wong TY, Donaghue KC et al (2012) Retinal arteriolar tortuosity is associated with retinopathy and early kidney dysfunction in type 1 diabetes. Am J Ophthalmol 153:176-183 
34. Kristinsson JK, Gottfredsdottir MS, Stefansson E (1997) Retinal vessel dilatation and elongation precedes diabetic macular oedema. Br J Ophthalmol 81:274-278

35. Sasongko MB, Wong TY, Nguyen TT, Cheung CY, Shaw JE, Wang JJ (2011) Retinal vascular tortuosity in persons with diabetes and diabetic retinopathy. Diabetologia 54:2409-2416

36. Tapp RJ, Williams $\mathrm{C}$, Witt $\mathrm{N}$ et al (2007) Impact of size at birth on the microvasculature: the Avon Longitudinal Study of Parents and Children. Pediatrics 120:e1225-e1228

37. Hartnett ME, Martiniuk D, Byfield G, Geisen P, Zeng G, Bautch VL (2008) Neutralizing VEGF decreases tortuosity and alters endothelial cell division orientation in arterioles and veins in a rat model of ROP: relevance to plus disease. Invest Ophthalmol Vis Sci 49:3107-3114

38. Gardiner TA, Archer DB, Curtis TM, Stitt AW (2007) Arteriolar involvement in the microvascular lesions of diabetic retinopathy: implications for pathogenesis. Microcirculation 14:25-38

39. Yim-Lui Cheung C, Wong TY, Lamoureux EL et al (2010) C-reactive protein and retinal microvascular caliber in a multiethnic asian population. Am J Epidemiol 171:206-213

40. Klein R, Klein BE, Knudtson MD, Wong TY, Tsai MY (2006) Are inflammatory factors related to retinal vessel caliber? The Beaver Dam Eye Study. Arch Ophthalmol 124:87-94

41. Daien V, Carriere I, Kawasaki R et al (2013) Retinal vascular caliber is associated with cardiovascular biomarkers of oxidative stress and inflammation: the POLA study. PLoS One 8:e71089

42. Rasmussen ML, Broe R, Frydkjaer-Olsen U et al (2017) Retinal vascular geometry and its association to microvascular complications in patients with type 1 diabetes: the Danish Cohort of Pediatric Diabetes 1987 (DCPD1987). Graefes Arch Clin Exp Ophthalmol 255:293-299

43. Antonetti DA, Klein R, Gardner TW (2012) Diabetic retinopathy. N Engl J Med 366:1227-1239

44. Ikram MK, Cheung CY, Lorenzi M et al (2013) Retinal vascular caliber as a biomarker for diabetes microvascular complications. Diabetes Care 36:750-759
45. Klein R, Klein BE, Moss SE et al (2004) The relation of retinal vessel caliber to the incidence and progression of diabetic retinopathy: XIX: the Wisconsin Epidemiologic Study of Diabetic Retinopathy. Arch Ophthalmol 122:76-83

46. Roy MS, Klein R, Janal MN (2011) Retinal venular diameter as an early indicator of progression to proliferative diabetic retinopathy with and without high-risk characteristics in African Americans with type 1 diabetes mellitus. Arch Ophthalmol 129:8-15

47. Cheung N, Donaghue KC, Liew G et al (2009) Quantitative assessment of early diabetic retinopathy using fractal analysis. Diabetes Care 32:106-110

48. Klein BE, Klein R, McBride PE et al (2004) Cardiovascular disease, mortality, and retinal microvascular characteristics in type 1 diabetes: Wisconsin epidemiologic study of diabetic retinopathy. Arch Intern Med 164:1917-1924

49. Lund SH, Aspelund T, Kirby P et al (2016) Individualised risk assessment for diabetic retinopathy and optimisation of screening intervals: a scientific approach to reducing healthcare costs. Br J Ophthalmol 100:683-687

50. Aspelund T, Thornorisdottir O, Olafsdottir E et al (2011) Individual risk assessment and information technology to optimise screening frequency for diabetic retinopathy. Diabetologia 54:2525-2532

51. Joshi V, Agurto C, VanNess R, Nemeth S, Soliz P, Barriga S (2014) Comprehensive automatic assessment of retinal vascular abnormalities for computer-assisted retinopathy grading. Conf Proc IEEE Eng Med Biol Soc 2014:6320-6323

52. Cavallari M, Stamile C, Umeton R, Calimeri F, Orzi F (2015) Novel method for automated analysis of retinal images: results in subjects with hypertensive retinopathy and CADASIL. Biomed Res Int 2015:752957

53. Gulshan V, Peng L, Coram M et al (2016) Development and validation of a deep learning algorithm for detection of diabetic retinopathy in retinal fundus photographs. JAMA 316:2402-2410

54. Wong TY, Bressler NM (2016) Artificial intelligence with deep learning technology looks into diabetic retinopathy screening. JAMA 316:2366-2367 